\title{
Metodologia de Organização de Dados Temporais para o Cadastro Territorial Multi- finalitário
}

\section{Temporal Data Organization Methodology for the Multipurpose Territorial Cadastre}

\author{
Glaucia Gabriel Sass ${ }^{1}$, Amilton Amorim ${ }^{2}$ e Rui Pedro Julião ${ }^{3}$
}

1 Universidade Estadual de Mato Grosso do Sul - UEMS, Curso de Ciência da Computação, Dourados, Mato Grosso do Sul, Brasil. glaucia@comp.uems.br

ORCID: https://orcid.org/0000-0002-0215-7816

2 Universidade Estadual Paulista "Julio de Mesquita Filho" - Campus Presidente Prudente, Departamento de Cartografia, Presidente Prudente - SP, Brasil. amorim@fct.unesp.br.

ORCID: https://orcid.org/0000-0002-4012-7079

3 Universidade de Nova Lisboa- UNL - Centro Interdisciplinar de Ciências Sociais (CICS.NOVA) - FCSH, Lisboa, Portugal. rpj@fcsh.unl.pt.

ORCID: https://orcid.org/0000-0002-5625-9965

Resumo: Atualmente, as mais diversas áreas da ciência buscam no passado dados para entender o presente e construir o futuro, indicando como a história é importante em nossas vidas. No Cadastro Territorial Multifinalitário (CTM) não é diferente, conhecer como eram os dados do CTM no passado pode indicar como houve a ocupação de um território e permite planejar como se desenvolverão as próximas ações de ocupação. O objetivo deste trabalho é apresentar uma metodologia para a organização de dados temporais para o CTM. A proposta metodológica consiste em uma série de etapas que, a partir do conceito de Data Warehousing, possibilitam organizar dados históricos, provenientes de diferentes fontes, unindo-os a dados atuais para gerar novas informações. Este método, aliado às ferramentas tecnológicas, permitiram unir em uma mesma base de dados, dados "diferentes" em sua estrutura e semântica, adquiridos em épocas distintas. Esse procedimento disponibiliza a história dos dados cadastrais, permitindo verificar como era, como é e fazer uma previsão do futuro daquele dado. A proposta metodológica se mostrou viável porque todas as tecnologias empregadas são livres e também porque as etapas são iterativas, ou seja, uma vez construído o modelo multidimensional, novas bases de dados podem ser inseridas no CTM temporal, baseado no Data Warehousing.

Palavras-chave: Cadastro Territorial Multifinalitário Temporal. Dados Espaço-Temporal. Data Warehousing.

\begin{abstract}
Nowadays, most diverse science areas search for past data to understand the present and build the future. In Multipurpose Territorial Cadastre (MTC), knowing the past can indicate in what manner there was an occupation of a territory also allowing one to plan on how to develop the next occupation actions. Our objective is to present a methodology for the organization of temporal data for the MTC. The methodological proposal consists of a series of steps that, from the concept of Data Warehousing, allows one to organize historical information from different sources, joining it to current data to generate new information. This method, along with the technological tools, allowed one to unite in a same database, "different" data in its structure and semantics acquired in different times. This procedure provides the history of the Cadastre data, and it is possible to verify what it was like, what it is like and allows a forecast of the future of that data. The methodological proposal proved viable because all the technologies employed are free and because the steps are iterative, once the multidimensional model is constructed, new databases can be inserted into the temporal.
\end{abstract}

Keywords: Temporal Multipurpose Cadastre. Spatio-Temporal Data. Data Warehousing.

\section{INTRODUÇÃO}

O Cadastro Territorial Multifinalitário (CTM), nas últimas décadas, sofreu grandes transformações conceituais e estruturais (AMORIM, 2018). Porém, em sua essência, continua objetivando gerenciar os dados relacionados às parcelas territoriais que podem ser de natureza diversa, se destacando como importante instrumento de suporte ao planejamento, à gestão territorial e ao desenvolvimento. 
A evolução do CTM está muito ligada à das tecnologias da informação que se aprimoraram e cresceram em recursos que podem ser utilizados para sua construção. Neste âmbito, destacam-se sugestões de gestão dos dados que, quando contextualizados, representam conhecimento e uma parte da história. No passado, os conjuntos de dados para CTM eram puramente descritivos, compostos por descrição textual e numérica. Com o desenvolvimento de novos equipamentos e técnicas, para aquisição, armazenamento e manipulação de dados é possível gerar informações com mais qualidade (posicional e temporal) e útil para os gestores.

Mesmo com o desenvolvimento de novas tecnologias, seu uso pelas mais diversas áreas do conhecimento não é imediato. Precisam ser elaboradas novas metodologias para agregar essas tecnologias aos processos estabelecidos e utilizados pelos profissionais consumidores dessas novas tecnologias.

Este trabalho propõe uma metodologia para representar a evolução do dado no tempo, com recursos de tecnologias aplicadas a outras áreas do conhecimento.

Desde o início da era tecnológica (em meados de século XX) os primeiros computadores começaram a processar e armazenar dados. Antigamente, o armazenamento de dados era feito por meio de cartões perfurados. Atualmente, com o avanço tecnológico, é possível armazenar dados em nuvens, ou seja, utiliza-se a arquitetura da Internet para armazenar dados e disponibilizar serviços aos usuários.

No contexto do CTM, assim como em outras áreas, os dados são adquiridos para representar as características do "objeto". Quando há a atualização dos dados que representam o objeto, o valor anterior do dado se perde, quando é sobrescrito. Exemplo: a área construída da parcela X em 21/02/2012 era 67,00 m2, na atualização cadastral de 2013 o valor da área construída aumentou para $97,00 \mathrm{~m} 2$, quando o valor 97 é inserido no Sistema Cadastral o valor 67 é apagado, e se perde. Isso acontece quando os Sistemas Cadastrais informatizados utilizam Sistemas Gerenciadores de Banco de Dados (SGBD) transacionais, que sobrescrevem o valor dos dados quando esses são atualizados.

Este trabalho tem como principal objetivo apresentar uma metodologia para o suporte do CTM utilizando dados de vários instantes temporais. Dessa forma, são apresentados recursos que possam recuperar dados antigos de Sistemas Cadastrais existentes e integrar esses dados históricos a novos dados para apoio à tomada de decisão.

\section{CADASTRO TERRITORIAL MULTIFINALITÁRIO}

Para Longley et al. (2013), "o Cadastro é definido como o mapa da propriedade da terra em uma área, mantido para fins de tributação da terra ou para a criação de um registro público de propriedade." Mas esta é uma acepção antiquada, pois desde o seu surgimento, os sistemas de cadastro passaram por várias fases, associando novas características conforme a evolução dos conceitos e necessidades da administração pública. Bennedett et al. (2011) apresenta como primeiros cadastros, o fiscal com o intuito de taxação territorial e o jurídico para a proteção do direito sobre o território e o desenvolvimento do comércio territorial. Refere-se ainda que nos anos de 1970 há o surgimento do Cadastro Multifinalitário, abordando também os conceitos ambientais, sociais e econômicos.

Williamson et al. (2013) apresenta a evolução do cadastro como estágios ao longo do tempo, são eles: Cadastro como ferramenta econômica e fiscal; Cadastro como ferramenta para transações imobiliárias; Cadastro como ferramenta para o planejamento; e por último Cadastro como ferramenta para o gerenciamento territorial. A última correspondendo ao CTM.

As transformações do Cadastro ocorreram por fatores que transformaram a sociedade como um todo. $\mathrm{Na}$ atualidade os fatores que influenciam os CTM são: a rápida urbanização, insegurança alimentar, mudança climática e informalidade do registro territorial (STEUDLER, 2014).

Para atender a esses preceitos, o CTM, geralmente, deve conter entidades geográficas e seus atributos, relacionados por um identificador único (ID), obedecendo a certas regras de forma que uma parcela esteja identificada inequivocadamente. Os dados podem ser organizados e baseados na parcela territorial, coletados, armazenados e referenciados (LONGLEY et al., 2013; WILLIAMSON et al., 2010; STEUDLER, 2014).

Os documentos textuais, arquivos literais, contêm dados descritivos das parcelas e das pessoas a elas relacionadas, por exemplo, identificador da parcela, nome do proprietário, área e uso. O mapa contém a 
representação cartográfica do levantamento sistemático das parcelas territoriais com elementos físicos e naturais.

A gestão do CTM é hoje efetuada utilizando Sistemas de Informação, apoiados em Sistemas de Informação Geográfica (SIG). Longley et al. (2013) consideram que os SIG são uma tecnologia de base tanto para a ciência quanto para a resolução de problemas "usando o conhecimento geral e o específico da realidade geográfica".

Os SIG ajudam a solucionar problemas complexos da realidade territorial e fornecem informações geográficas para a tomada de decisão em inúmeras áreas, como na gestão de saúde, empresas de entrega de mercadorias, gestão de rodovias, companhias de silvicultura, gestão de parques, planejamento de viagens e turismo (LONGLEY et al., 2013). Por tanta diversidade de aplicações, os SIG recebem diferentes denominações de acordo com a área de aplicação e o tipo de dados que administram.

Os SIG, orientados para o CTM, com aquisição, processamento, armazenamento e distribuição de informações sobre o território, com base na parcela territorial, são comumente denominados de Sistema de Informação Territorial (SIT) (PIUMETTO; ERBA, 2007; STEUDLER, 2014).

Para Williamson et al. (2010) o Cadastro é um componente fundamental para os Sistemas de Administração Territorial (Land Administration Systems - LAS), que fornecem descrição de como as pessoas se relacionam com o território e sua propriedade.

Os SIT fornecem os dados na forma de um produto (como mapas ou títulos certificados) ou em forma de serviços (como aplicativos). Estes sistemas integram dados de atributos que podem ser apresentados de forma textual ou numérica, dados espaciais que podem ser apresentados em mapas, e dados temporais que indicam sua ocorrência (DALE; MCLAUGHLIN, 1990). Liu et al. (2019) apresenta essa composição com três dimensões: a espacial, temporal e as propriedades do objeto em si.

Polat et al. (2018) apresentam um estudo de caso baseado no modelo RRR (Rights, Restrictions and Responsibilities) cujo principal objetivo foi a modelagem de direitos de servidão em um estudo de caso na Turquia. A modelagem proposta inclui atributos temporais aos dados cadastrais relacionados às servidões, incorporados ao chamado "Pacote Administrativo", demonstrando que a modelagem LADM (Land Administration Domain Model) pode ser usada para descrever a dimensão temporal do dado cadastral, mas que existem diferenças semânticas, semelhanças e incompatibilidade de classes e atributos entre os princípios estabelecidos pela LADM e o sistema de informação cadastral, para o caso analisado na Turquia.

Há que se destacar a utilização da LADM que, de acordo com Amorim et al. (2018), facilita o desenvolvimento de projetos de gestão territorial e de softwares utilizados para tanto, no sentido de contribuir com as boas práticas na administração pública, com responsabilidades nesse domínio e assim contribuindo também para o desenvolvimento sustentável.

A Figura 1 apresenta uma forma de representar os dados espaciais e temporais das parcelas territoriais em um SIT, onde se observa a agregação e o fracionamento das parcelas (SASS; AMORIM, 2013).

Pode-se dizer que os SIT são ferramentas para apoiar o processo de tomada de decisão, seja em um projeto ou em uma instituição, que agregam os dados geográficos e as bases de dados alfanuméricos para gerar uma visão mais completa e adequada da realidade analisada (PIUMETTO; ERBA, 2007). Daí a necessidade de contemplar os dados temporais de forma adequada. 
Figura 1 - Representação de Dados Espaciais e Temporais.

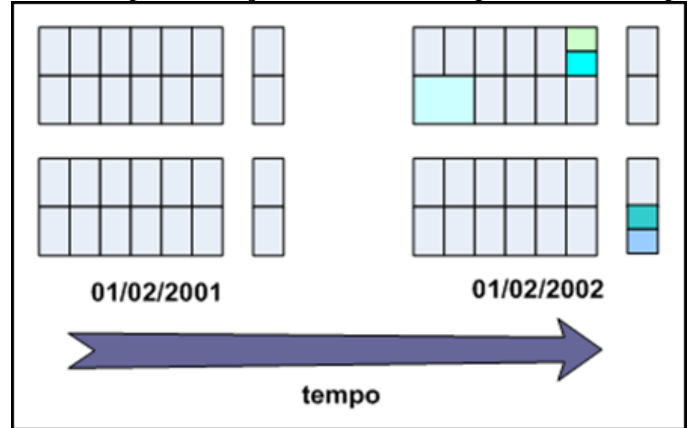

Fonte: Sass e Amorim (2013).

Nos SIT os dados são armazenados em Sistemas SGBD. Os SGDB tradicionais (ou transacionais), em sua maioria, armazenam dados alfanuméricos e são comumente chamados de banco de dados convencionais (exemplo: inteiro, data, reais e texto). Quando outros tipos de dados são armazenados, como dados geográficos e dados temporais, os bancos de dados são chamados não convencionais. Para o armazenamento desses diferentes tipos de dados, espaciais e temporais, novas tecnologias para banco de dados foram desenvolvidas nos últimos anos, levando em consideração as suas peculiaridades (SASS, 2013).

\section{DADOS CADASTRAIS}

Tal como referido anteriormente, os elementos fundamentais dos SGBD são os dados, podendo ser convencionais ou não convencionais. Os dados convencionais, sem considerar o seu estado (tempo) fazem parte do dia a dia das organizações e são bem conhecidos. No entanto, os dados temporais ainda são objetos de muitas pesquisas, principalmente se tratando dos dados cadastrais.

Os dados convencionais representam os eventos do mundo real. Os dados temporais complementam os dados convencionais, representam os eventos do mundo real marcados com o tempo. Para definir quais eventos do mundo real serão registrados com o tempo de ocorrência é necessário fazer algumas considerações sobre o tempo. Essas considerações partem da definição de várias características temporais: dimensão temporal, ordem no tempo, tempo absoluto e tempo relativo, granularidade no tempo, variação temporal, instante no tempo, intervalo de tempo, elemento temporal e duração temporal. De acordo com Jensen et al. 1998, esses conceitos básicos sobre o dado temporal foram reunidos no Glossary of Temporal Database (Glossário de Banco de Dados Temporal), que representa a intenção de unificação dos conceitos ligados aos Banco de Dados Temporais - BDT. Estas características serão descritas a seguir:

a) dimensão temporal: os modelos de dados temporais possuem uma dimensão (dimensão temporal) a mais do que os modelos de dados convencionais;

b) ordem no tempo (Figura 2): ordem linear, no qual um ponto no tempo terá somente um antecessor e um predecessor; ordem ramificada, permite que um ponto no tempo tenha mais de um antecessor e mais de um predecessor; ordem circular, modela processos recorrentes, como datas comemorativas, mas não é o tempo que se repete e sim os eventos;

Figura 2 - Tipos de Ordem Temporal.

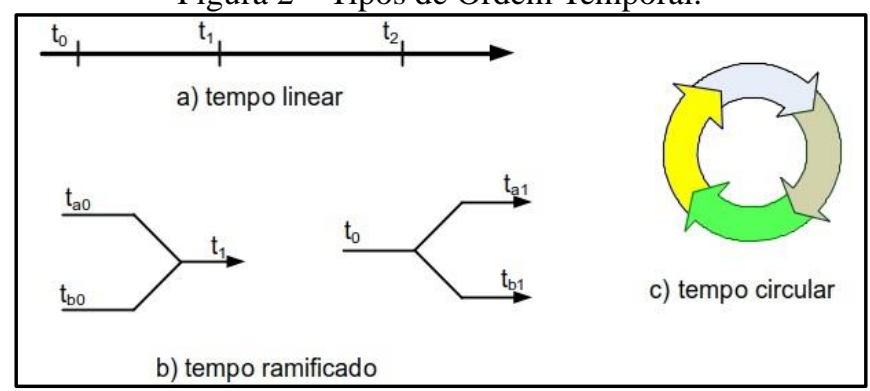

Fonte: Sass (2013). 
c) tempo absoluto e tempo relativo: o absoluto indica que um tempo válido considera a granularidade associada com o fato, enquanto o relativo indica que o tempo válido de um fato está relacionado há algum outro tempo;

d) granularidade no tempo: tamanho da unidade de tempo considerada no sistema, pode ser hora, dia, mês ou ano;

e) variação temporal: pode ser representado pelo tempo contínuo (entre dois instantes consecutivos temporais pode existir outro instante temporal) ou tempo discreto (baseado em uma linha de tempo composta de uma sequência de intervalos temporais consecutivos que não podem ser decompostos, de mesma duração);

f) instante no tempo: representado por um chronon no eixo temporal do modelo, para o tempo discreto;

g) intervalo de tempo: possui tempo de início (s) e tempo de término (e), definindo o conjunto de todos os tempos $(\mathrm{t})$, tal que $\mathrm{s} \leq \mathrm{t} \leq \mathrm{e}$;

h) elemento temporal: união finita de intervalos de tempo; e

i) duração temporal: dependente do contexto, pode ser fixa ou variável.

Para Medeiros e Jomier (1993) um SIG acrescido do componente tempo permite prever o futuro baseado em registros do passado, analisar a evolução temporal dos dados, analisar comparativamente cenários simulados e analisar de forma comparativa os dados atuais e os cenários simulados.

Consequentemente, o SIT torna-se um instrumento para o gerenciamento do território, fornecendo aos gestores informações históricas, disponibilizadas em mapas temáticos que permitem a análise visual das ocorrências registrada em sua base de dados.

Um aspecto importante sobre os produtos gerados por SIT é permitir a análise visual sobre as informações oferecidas pelo CTM temporal. A análise visual fomenta o desenvolvimento de conhecimento, métodos, tecnologias e práticas que exploram e combinam pontos fortes de processos humanos e de processamento de dados eletrônico (KEIM et al., 2008; ANDRIENKO et al., 2010)

A análise visual combina técnicas de análise automatizada com visualizações interativas para uma compreensão efetiva, inferência e tomada de decisão com base em grandes e complexos conjuntos de dados (KEIM et al., 2008).

Para Keim et al. (2008) o objetivo da análise visual é criar ferramentas e técnicas para permitir que as pessoas: (a) sintetizem informações para obter entendimento sobre dados massivos, dinâmicos, ambíguos e muitas vezes conflitantes; (b) detectem o esperado e descubram o inesperado; (c) realizem avaliações em tempo útil, justificáveis e compreensíveis; (e) comuniquem de forma eficaz a avaliação para a ação.

A análise visual apresenta diferentes soluções para um problema, deixando que o usuário interprete essas opções no contexto da aplicação e gere conhecimento adequado para que ele possa discutir com seus pares as melhores soluções.

\section{TECNOLOGIAS PARA DADOS TEMPORAIS}

Atualmente, duas tecnologias se destacam para o tratamento de dados temporais, Banco de Dados Temporal e Data Warehouse. Ambas possuem características específicas que precisam ser avaliadas para que se possam escolher, entre elas, qual utilizar. A seguir, apresenta-se a descrição dessas tecnologias.

\subsection{Banco de Dados Temporal (BDT)}

Date (2000) considera que um BDT "é aquele que contém dados históricos em vez de, ou além de, dados atuais. [...] os dados [...] são apenas inseridos, nunca eliminados ou atualizados", que difere do banco de dados transacional "que contém apenas dados atuais porque os dados anteriores foram eliminados". 
No BDT o tempo é diferenciado em tempo medido pelo sistema (transação) e o tempo observado no mundo real (válido). O tempo de transação é gerado automaticamente pelo sistema para um evento a partir da data e hora atual do computador. Representa o intervalo de tempo durante o qual o evento é atual (tempo presente) no sistema de banco de dados. O tempo válido para um evento é o conjunto de intervalos de tempo durante o qual o evento é verdadeiro no mundo real, não pode ser gerado automaticamente pelo sistema, mas deve ser fornecido ao sistema (SILBERSCHATZ et al., 2006).

No BDT uma relação temporal é aquela em que cada instância de entidade possui um tempo associado, que pode ser o tempo válido ou o tempo de transação. Em um BDT podem ser armazenados os dois tipos de tempo, válido ou de transação, nesse caso as relações são consideradas bitemporais (SILBERSCHATZ et al., 2006).

Para projetar um BDT o modelo de dados deve permitir a definição e representação do dado temporal. O modelo conceitual temporal precisa ser de fácil compreensão, compatível com modelos e aplicações anteriores e, principalmente, permitir ao projetista do Banco de Dados mesclar os aspectos temporais e não temporais, pois uma aplicação pode não ser inteiramente temporal (JENSEN; SNODGRASS, 1999).

Segundo Edeweiss (1998) para analisar um modelo de dados temporais alguns aspectos precisam ser considerados:

a) identificar o tipo de rótulo temporal utilizado pelo modelo (ponto no tempo, intervalo temporal, elemento temporal, duração);

b) analisar a forma de variação temporal dos atributos;

c) verificar se os rótulos temporais são explícitos ou implícitos;

d) homogeneidade temporal;

e) apresentação e funcionalidades da linguagem de consulta.

O padrão da Linguagem de Consulta Estruturada (Structured Query Language - SQL) define os tipos date, time, timestamp e interval. $\mathrm{O}$ tipo date contém quatro dígitos para o ano, dois dígitos para o mês e dois dígitos para o dia. O tipo time contém dois dígitos para a hora, dois dígitos para o minuto e dois dígitos para o segundo, mais dígitos fracionários opcionais. O tipo timestamp contém os campos de data e hora, com seis dígitos fracionários para o campo de segundos. O tipo interval permite referir a um período de tempo como "1 dia" ou "2 dias e 3 horas", sem especificar determinada hora quando esse período começa. O SQL, até a versão 2003, não forneceu nenhum suporte especial para dados temporais, além dos tipos de dados e operações relacionadas a tempo (SILBERSCHATZ et al., 2006).

Concomitantemente com as pesquisas sobre BDT, um grande esforço tem sido realizado com o objetivo de definir uma linguagem de consulta temporal. A partir de discussões envolvendo pesquisadores de diversos países surgiu a Temporal Structured Query Language - TSQL2 (Quadro 1), uma extensão da SQL1992 (SNODGRASS, 1995).

Quadro 1 - TSQL2 - Características relevantes.

\begin{tabular}{|l|l|}
\hline \multicolumn{1}{|c|}{ Característica } & \multicolumn{1}{c|}{ Descrição } \\
\hline Relacional & Consistente com o padrão SQL, especialmente com o SQL-1992 \\
\hline Compreensível & $\begin{array}{l}\text { Construída, estendida de maneira natural, a qual suporta todas as funcionalidades da SQL, } \\
\text { incluindo atualização, agregações e especificação e evolução de esquema. Suportará tempo de } \\
\text { validade e transação }\end{array}$ \\
\hline Álgebra associada & $\begin{array}{l}\text { Existência de um executável equivalente para a construção declarativa na linguagem, e mostrará } \\
\text { estratégias de implementação }\end{array}$ \\
\hline Linguagem de projeto & $\begin{array}{l}\text { Não define estrutura de armazenamento, estruturas de indexação, suportar sistemas distribuídos, } \\
\text { Banco de Dados heterogêneos ou técnicas de otimização }\end{array}$ \\
\hline Áreas de convergência & $\begin{array}{l}\text { Evita áreas ativas de pesquisa nas quais novos resultados são gerados frequentemente. Tais áreas } \\
\text { incluem suporte para recursão e projeto de BDT }\end{array}$ \\
\hline
\end{tabular}

Fonte: Adaptado de Snodgrass (1995).

Snodgrass (1995) refere que a TSQL2 deverá suportar: 
a) diferentes períodos de tempo. Em SQL somente date, time, timestamp e interval;

b) múltiplas granularidades, incluindo semestre, semana, estação de ano, etc. Em SQL somente existe year, month, day, hour, minute e second;

c) múltiplas linguagens, como por exemplo, 15 de maio de 2011;

d) múltiplos calendários, como por exemplo, lunar, acadêmico, fiscal e eras geológicas;

e) tempo indeterminado, como por exemplo, entre 2 e 10 de maio;

f) suporte a tempo histórico.

Yuan et al (2017) utilizam os recursos da TSQL2 para armazenar e recuperar informações históricas dos dados registrados no processo de construção, eles tratam, no trabalho, como dados dinâmicos e dados estáticos.

Para além da TSQL2 há outras propostas de linguagens de consulta temporal desenvolvidas, considerando a inserção da dimensão temporal ao padrão SQL. O SQL/Temporal foi proposto como uma parte do padrão SQL:1999 e seus conceitos e estruturas foram incluídas no SQL:2011 Standard (DEBELJAČKI, BOŠNJAK e ŠEREŠ, 2015). Muitas de suas facilidades têm sido incorporadas por SGBD comerciais como, Oracle e o PostgreSQL. Assim, o SQL/Temporal é uma linguagem de definição apropriada para dar suporte temporal às stored procedures.

No contexto das bases de dados, duas dimensões de tempo são importantes: o tempo de validade e o tempo de transação (SNODGRASS et al., 2012). Na sequência um relato dessas características no PostgresSQL e no Oracle.

O PostgreSQL é um SGBD objeto relacional de código fonte aberto, descendente do Postgre, cujo nome é derivado do Ingres, ambos desenvolvidos pelo Professor Michael Stonebraker da Universidade da Califórnia, em Berkeley. Ele oferece recursos como consultas complexas, chaves estrangeiras, triggers, views, integridade transacional e controle de concorrência de múltiplas versões (AILAMAKI et al., 2006).

O PostgreSQL 9.5.2 suporta o conjunto completo dos tipos de data e tempo do padrão SQL, timestamp, date, time e interval (PGDG 2016). Baseado no padrão SQL: 2011, o PostgreSQL, por meio da extensão temporal table, permite a criação e manipulação de tabelas temporais (DAVE, 2015).

A Oracle foi a primeira empresa a disponibilizar comercialmente um Banco de Dados relacional. Além do servidor de Banco de Dados, ela oferece diversas ferramentas diretamente relacionadas ao desenvolvimento e gerenciamento de Banco de Dados, ferramentas de inteligência de negócios e servidor de aplicação (JAKOBSSON e ORACLE, 2006).

Estão disponíveis ferramentas para projeto de Banco de Dados, consulta, geração de relatório e análise, incluindo o On-Line Analytical Processing (OLAP). Com o objetivo de reduzir a complexidade dos aspectos de criação e administração de um Banco de Dados, um esforço foi empregado na versão Oracle $10 \mathrm{~g}$, permitindo a criação de Banco de Dados, ajuste, gerenciamento de espaço, gerenciamento de memória, diagnósticos de desempenho e gerenciamento de carga de trabalho (JAKOBSSON; ORACLE, 2006).

O Oracle $10 \mathrm{~g}$ possui suporte para tabelas com tempo de validade, tabelas com tempo de transação, tabelas bitemporais, chaves primárias sequenciadas, unicidade sequenciada, integridade referencial sequenciada, e seleção e projeção sequenciada de um modo muito semelhante ao proposto no SQL/Temporal. Ao Oracle 11g foi acrescentado suporte avançado para consultas em tempo de validade (ORACLE, 2013).

\subsection{Data Warehouse}

O Data Warehouse (DW) ou o Data Warehousing é um dos componentes do conceito de Inteligência de Negócio (Business Intelligence - BI). Para Rob e Coronel (2011) o BI é um amplo, coeso e integrado conjunto de ferramentas e processos usados para adquirir, integrar, armazenar e analisar dados para a geração e apresentação de informações que deem apoio à tomada de decisões em organizações. Ele não é simplesmente uma ferramenta, mas um modelo de conceitos, práticas, ferramentas e tecnologias que ajudam uma organização a compreender melhor seus recursos centrais, fornecem um retrato "instantâneo" da situação e identificam oportunidades. 
O DW é um tipo particular de banco de dados. Eles surgiram para atender a necessidade de fornecer uma origem de dados única, limpa e consistente para apoio à decisão e de fazê-lo sem impactar os sistemas operacionais (DATE, 2000). São otimizados para recuperar dados e não processar transações. São diferentes dos bancos de dados convencionais em estrutura, funcionalidade, desempenho e propósito (ELMASRI; NAVATHE, 2005).

Inmon (2005) define o termo Data Warehouse como "um conjunto de dados baseado em assuntos, integrado, não volátil e variável em relação ao tempo de apoio às decisões gerenciais". A característica "variável no tempo" representa os dados em um fluxo através do tempo.

O principal objetivo do DW é disponibilizar informações de apoio à decisão em organizações. O DW é uma arquitetura, não uma tecnologia. Ele proporciona uma integração dos dados de uma instituição para a realização de análises estratégicas. Preocupa-se em integrar e consolidar os dados de fontes internas, provavelmente, heterogêneas e fontes externas, sumarizando, filtrando e limpando esses dados, preparando-os para a análise e o apoio à tomada de decisão.

A definição do DW tradicional trata a variável tempo como uma de suas características, porém ele não a gerencia como se propõe nos BDT. Os DW tradicionais e sistema OLAP não permitem a evolução de dados nas Dimensões (VAISMAN; ZIMÁNYI, 2009). Por isso, agregando os dados espaciais e temporais foi criado o conceito de Data Warehouse Espaço-Temporal (Spatio-Temporal Data Warehouse - STDW) para tratar as especificidades dos dados temporais (VAISMAN; ZIMÁNYI, 2019).

O STDW é um conjunto de dados baseado em assuntos, integrado, não volátil e variável em relação ao tempo, para apoio às decisões gerenciais, como definido por Inmon (2005) acrescido de dados espaciais e temporais. A literatura apresenta o STDW como a união do DW espacial/geográfico (Spatial DW - SDW) e o DW temporal (Temporal DW - TDW) (VAISMAN; ZIMÁNYI, 2019).

Fonseca et al. (2007) definem um SDW como uma extensão da abordagem tradicional de DW, acrescentando um componente geográfico. Basicamente, inclui no Modelo Estrela as propriedades geográficas (descritivas e geométricas), as quais podem ser definidas como Dimensões e/ou Medidas do SDW. As Dimensões podem armazenar as geometrias e as descrições dos objetos geográficos, enquanto as medidas espaciais armazenam apenas as geometrias.

Para o desenvolvimento do um STDW é preciso criar um modelo espaço-temporal multidimensional, importante para o suporte dessas aplicações, mas, a definição desse modelo deve considerar questões específicas de cada domínio (MALINOWSKI; ZIMÁNYI, 2008).

O modelo MultiDim, publicado em 2008, desenvolvido por Malinowski e Zimányi (2008) apresenta um modelo para o desenvolvimento de DW, além de considerar o espaço e o tempo. Ele permite uma representação no nível conceitual de todos os elementos requeridos no DW e aplicações OLAP, por exemplo, Dimensões, Hierarquia e Fatos associados às Medidas, com algumas extensões. São definidos os modelos de alto nível e do nível intermediário de acordo com a classificação de Inmon (2005).

O trabalho de Vaisman e Zimányi (2019) estende o modelo MultiDim para o uso em Mobility Data Warehouses, DW Móvel, apresentando a abrangência, o volume de dados que estão sendo gerado e a necessidade de integração desses dados.

Trajcevski et al. (2015), também, apresenta um estudo sobre a trajetória de pontos de interesse utilizando DW, e estende seu estudo aos objetos espaciais que variam ao longo do tempo em STDW. Eles definem os DW de Trajetória (TDW) como um caso particular de STDW, onde trajetórias fazem parte do DW, seja como dimensões ou medidas.

\section{PROCEDIMENTOS METODOLÓGICOS}

Esta seção descreve a metodologia para o desenvolvimento do CTM temporal que permite a criação de um sistema de apoio à tomada de decisão, considerando componentes espaço-temporais para o CTM. O método para a organização de dados temporais para o CTM é composto pelas etapas apresentadas na Figura 3. 
Na primeira etapa do método são definidas as fontes de dados utilizadas para a elaboração do STDW. Essas fontes de dados podem ser banco de dados convencionais, banco de dados geográficos, cópias de segurança de bancos de dados, arquivos texto e/ou arquivos de planilhas eletrônicas.

Figura 3 - Etapas do Método de Análise Temporal para CTM.

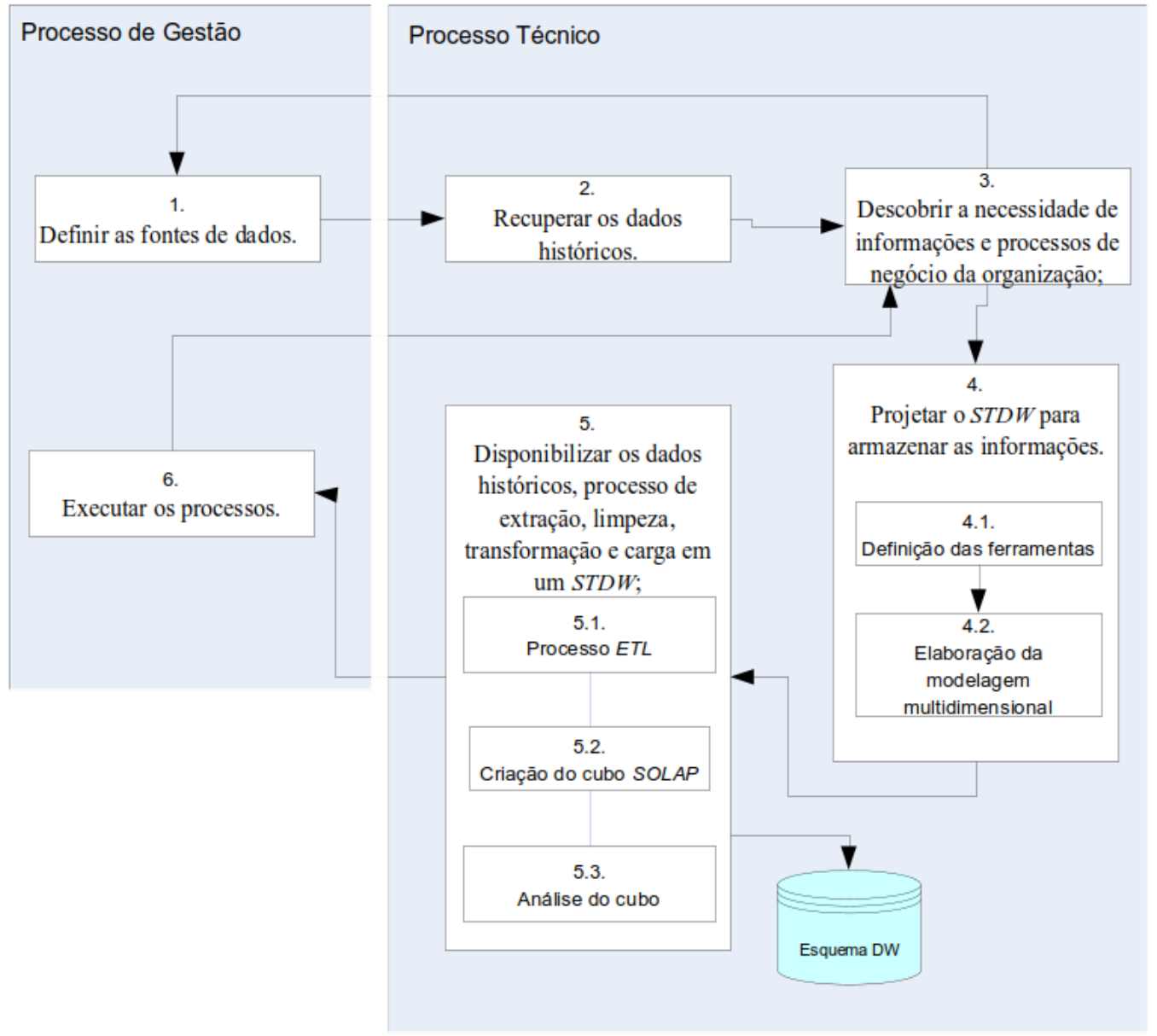

Fonte: Sass (2013).

Na segunda etapa, os dados são restaurados em seus aplicativos de origem (SGBD, planilha eletrônica, editor de texto, etc.) para a verificação de quais dados existem e como estão organizados. A partir dessa atividade é possível descrever, relacionar os dados existentes e definir os metadados do STDW.

$\mathrm{Na}$ terceira etapa são identificados, com base nos dados recuperados, as necessidades de informações e indicadores de negócio para as tomadas de decisões da organização. Para apoiar a execução dessa etapa, uma descrição das fontes de dados deve ser elaborada, gerando os metadados individuais do CTM. Em algumas situações, no final dessa etapa, pode ser identificada a necessidade de se trabalhar com outras fontes de dados. Se for o caso, volta-se para a etapa 1.

Para a definição dos metadados precisam ser considerados os conceitos de integração de dados. A integração de dados é um desafio para aplicações que precisam realizar consultas em várias fontes de dados independentes e heterogêneas (SASS, 2013). Por isso, nesta metodologia, a heterogeneidade foi classificada segundo os critérios apresentados por Sheth (1999), pois foi necessário integrar dados antigos aos novos dados no STDW.

A partir das informações resultantes da etapa três são descritos os processos de negócio para o projeto do STDW (quarta etapa) responsável pelo armazenamento das informações. A quarta etapa é composta por duas subetapas: subetapa 4.1, são escolhidas as ferramentas; subetapa 4.2, é elaborado o modelo multidimensional.

Na quinta etapa são disponibilizados para uso do STDW os dados históricos recuperados na etapa dois. Na subetapa 5.1 é executado o processo de extração, limpeza, transformação e carga dos dados. A subetapa 
5.2, é responsável pela criação do cubo do DW. Com o STDW carregado, os processos de consultas são disponibilizados para as análises, e na subetapa 5.3, diversos relatórios são gerados.

Na etapa seis o Data Warehousing é disponibilizado para os testes. Com a utilização das informações geradas, novas necessidades provavelmente surgem. Dessa forma, o processo volta para a etapa três, para verificar se novas fontes de dados são necessárias, caso não seja, o processo continua. O método se torna iterativo, para atender às necessidades dos usuários e para o refinamento dos dados.

\section{RESULTADOS}

Seguindo as etapas apresentadas na Figura 3, foi elaborado um CTM temporal para um pequeno município, baseado dos conceitos de STDW. Esse município possuía o backup de bancos de dados de antigas aquisições e um novo sistema cadastral com BDT estava sendo implementado. As etapas foram desenvolvidas como seguem:

a) Etapa 1: as fontes de dados escolhidas estavam armazenadas em Paradox 4.0, MS ACESS 2000, PostgreSQL 8.4 e PostgreSQL 9.1;

b) Etapa 2: os dados foram restaurados em seus bancos de dados de origem para a verificação de quais dados existiam e como estavam organizados. A partir dessa atividade foi possível descrever e relacionar os dados existentes nos quatro levantamentos e definir os metadados do STDW;

c) Etapa 3: uma descrição das fontes de dados foi elaborada, gerando os metadados individuais de cada base de dados. Com base nessas descrições, foram definidas as informações que seriam geradas;

d) Etapa 4.1: as ferramentas escolhidas são livres, PostgreSQL 9.1, Postgis 1.5, gvSIG 1.11 e a suíte Pentaho 4.8;

e) Etapa 4.2: o modelo multidimensional foi elaborado na ferramenta Case e diagramado no ArgoCaseGeo 3.0. Essa ferramenta é utilizada para a modelagem orientada a objetos, no entanto, foi empregada para a modelagem relacional por oferecer os estereótipos de tempo e espaço para a diagramação;

f) Etapa 5.1: foi utilizada a ferramenta ETL GeoKettle para extração, limpeza, transformação e carga dos dados;

g) Etapa 5.2: foi criado o cubo no Schema Workbench 3.5;

h) Etapa 5.3: os processos de consultas foram disponibilizados para as análises, no qual diversos relatórios foram gerados com o Report Designer 3.9.1-GA. O Metadata Editor 4.8 foi utilizado para a geração do metamodelo que permite ao gestor gerar os próprios relatórios;

i) Etapa 6: o Data Warehousing foi disponibilizado para os testes no BI Server e no gvSIG.

Como resultado do processo, foram disponibilizados uma série de relatórios textuais, gráficos e mapas com dados temporais. Um exemplo de relatório gerado é mostrado na Figura 4, sobre residentes, com as ocorrências referentes às parcelas, nos anos de 1996, 2004, 2010 e 2012. 
Figura 4 - Relatório de Residentes Desenvolvido no ReportDesign.

\begin{tabular}{|c|c|c|c|c|c|c|c|c|c|c|c|c|}
\hline \multirow[b]{2}{*}{ SSQQLLFF } & \multirow[b]{2}{*}{ Homens } & \multicolumn{7}{|c|}{$\begin{array}{l}\text { Residentes nas Parcelas } \\
\text { Anos 1996, 2004, 2010 e } 2012\end{array}$} & \multirow[b]{2}{*}{$\begin{array}{l}\text { De } 22 \text { a } \\
45 \text { anos }\end{array}$} & \multirow[b]{2}{*}{$\begin{array}{l}\text { De } 46 \text { a } \\
60 \text { anos }\end{array}$} & $1 / 119$ & \multirow[b]{2}{*}{ Ano } \\
\hline & & Mulheres & $\begin{array}{c}\text { Até } 1 \\
\text { ano }\end{array}$ & $\begin{array}{l}\text { De } 1 \text { a } \\
3 \text { anos }\end{array}$ & $\begin{array}{l}\text { De } 4 \text { a } \\
6 \text { anos }\end{array}$ & $\begin{array}{l}\text { De } 7 \text { a } \\
9 \text { anos }\end{array}$ & $\begin{array}{l}\text { De } 10 \text { a } \\
15 \text { anos }\end{array}$ & $\begin{array}{l}\text { De } 16 \text { a } \\
21 \text { anos }\end{array}$ & & & $\begin{array}{l}\text { Mais de } \\
60 \text { anos }\end{array}$ & \\
\hline \multicolumn{13}{|l|}{1020101} \\
\hline & 2 & 2 & & & & & & & 1 & 1 & & 1996 \\
\hline & 0 & 1 & 0 & 0 & 0 & 0 & 0 & 0 & 0 & 1 & & 2004 \\
\hline & 2 & 3 & 0 & 0 & 1 & 0 & 0 & 0 & 2 & 1 & 0 & 2010 \\
\hline \multicolumn{13}{|l|}{1020102} \\
\hline & 3 & 1 & & & & & 2 & & 1 & 1 & & 1996 \\
\hline \multicolumn{13}{|l|}{1020103} \\
\hline & 2 & 2 & & & 1 & & 1 & & 2 & & & 1996 \\
\hline \multicolumn{13}{|l|}{1020104} \\
\hline & 4 & 1 & & 1 & 1 & & 1 & & 2 & & & 1996 \\
\hline \multicolumn{13}{|l|}{1020201} \\
\hline & 1 & 3 & & & & 1 & 1 & & 2 & & & 1996 \\
\hline & 1 & 3 & 0 & 0 & 0 & 0 & 1 & 1 & 2 & 0 & & 2004 \\
\hline & 1 & 3 & 0 & 0 & 0 & 0 & 0 & 1 & 2 & 1 & 0 & 2010 \\
\hline & 1 & 3 & 0 & 0 & 0 & 0 & 0 & 0 & 2 & 2 & 0 & 2012 \\
\hline \multicolumn{13}{|l|}{1020301} \\
\hline & 1 & 1 & & & & & & & & 2 & & 1996 \\
\hline & 0 & 1 & 0 & 0 & 0 & 0 & 0 & 0 & 0 & 1 & & 2004 \\
\hline & 1 & 1 & 0 & 0 & 0 & 0 & 0 & 0 & 1 & 1 & 0 & 2010 \\
\hline \multicolumn{13}{|l|}{1020401} \\
\hline & 1 & 3 & 0 & 0 & 0 & 0 & 0 & 0 & 3 & 2 & 0 & 2012 \\
\hline
\end{tabular}

Fonte: Os autores (2018).

A Figura 5 apresenta um mapa temático com dados espacializados sobre a ocorrência de cardiopatia nos residentes das parcelas, considerando os quatro anos da amostra. No caso dos relatórios gráficos, um exemplo é apresentado na Figura 6. A Figura 7, apresenta uma representação gráfica, na qual poder ser observado o fracionamento de algumas parcelas, ocorrido entre os anos de 2010 e 2012. Este tipo de análise só é possível por meio da manutenção dos dados temporais.

Figura 5 - Mapa da Ocorrência de Cardiopatia em 1996, 2004, 2010 e 2012.

Parcelas que Representam a Ocorrência dos Casos de Cardiopatia

nos Levantamentos de 1996, 2004, 2010 e 2012

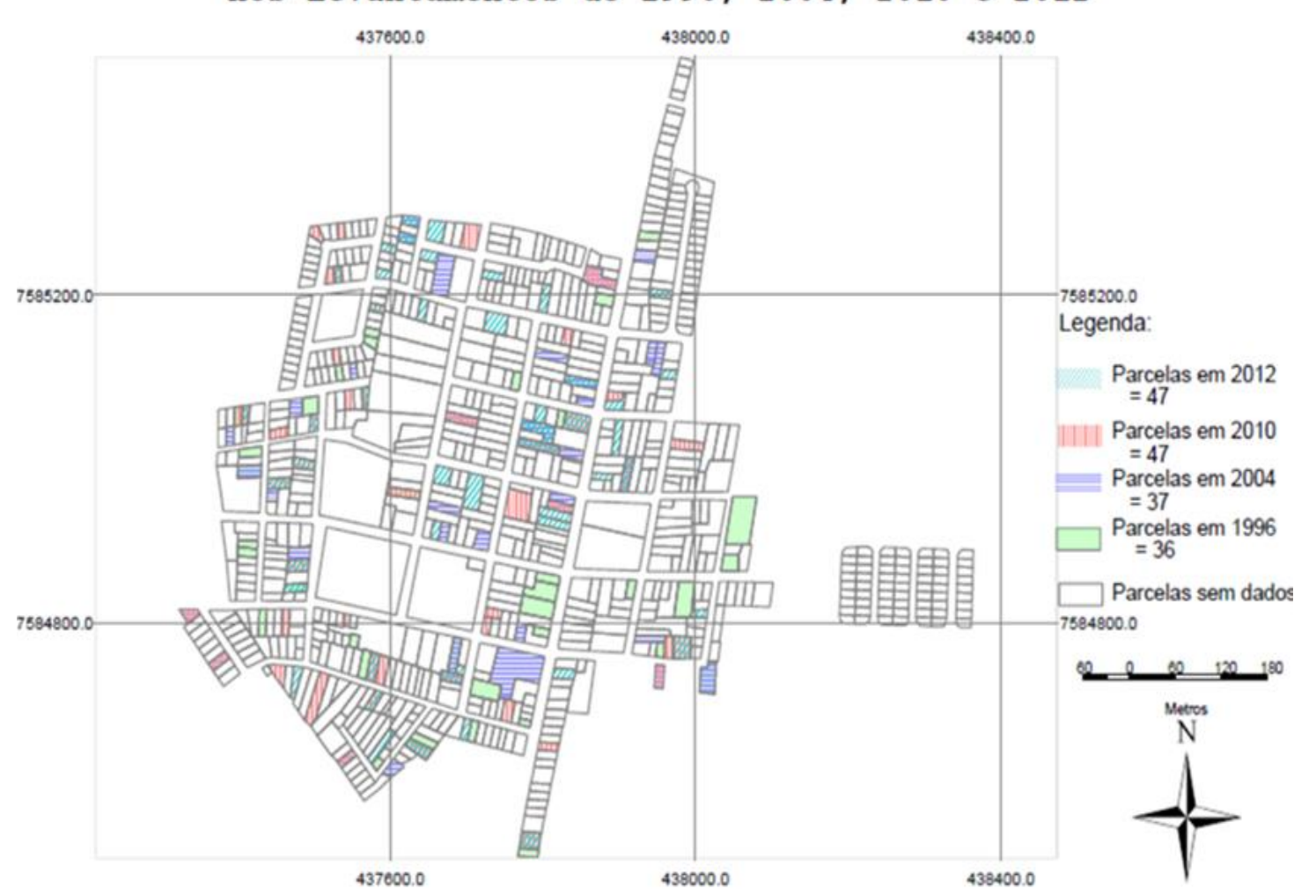

Fonte: Os autores (2018). 
Figura 6 - Gráfico de Evolução de Patologias ao Longo do Tempo.

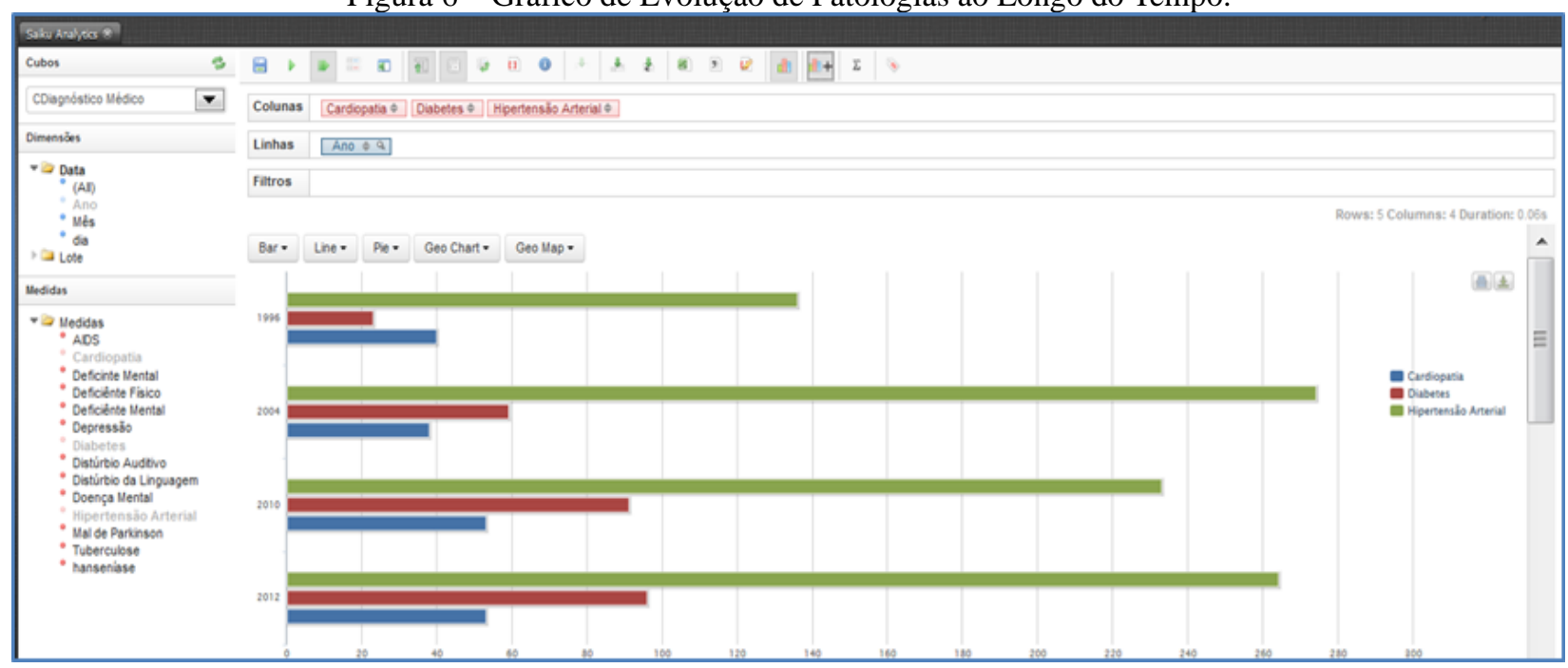

Fonte: Os autores (2018).

Figura 7 - Mapa com a Consulta SQL Sobre Desmembramento entre 2010 e 2012.

Parcelas que Representam Desmembramentos entre

os Levantamentos de 2010 e 2012

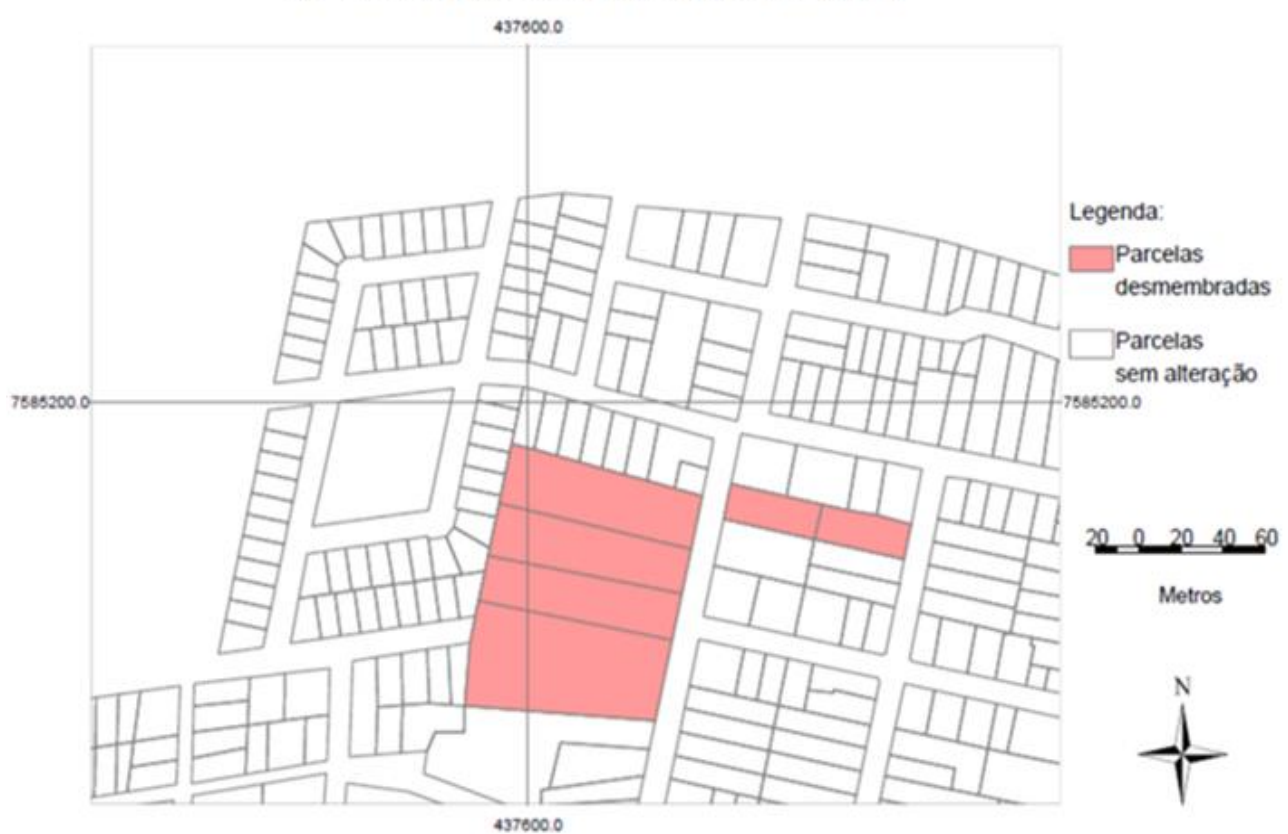

Fonte: Os autores (2018).

\section{DISCUSSÕES E CONCLUSÕES}

O CTM, de maneira geral, precisa de uma administração efetiva que abranja todas as suas características em benefício do próprio município e melhoria da qualidade de vida da população. Dessa forma, considerando os problemas de integração de dados, evolução histórica e disponibilização dos dados do CTM, para o desenvolvimento deste método, o trabalho foi baseado no processo de BI, utilizando Data Warehousing, no qual dados cadastrais advindos de fontes e épocas distintas, fornecem ao administrador público informações para o apoio à tomada de decisão.

Ao longo deste trabalho, identificou-se que o aspecto tempo não é um elemento novo na elaboração do CTM, sendo um elemento importante para várias atividades, mas que geralmente não é implementado adequadamente. Sabe-se que o histórico de dados é importante para entender a evolução do cadastro das parcelas, como apresentado na Figura 7, ou de eventos que a elas podem estar associados, mas, atualmente, após cada aquisição e atualização, ocorre uma substituição desses dados, sendo eliminados os anteriores. Por 
isso, identificar e organizar os dados antigos para integrá-los aos novos dados cadastrais é importante para recuperar a história de diversos fatos ocorridos.

Dessa forma, a recuperação dos dados de levantamentos anteriores permite elaborar um retrato da evolução de várias situações importantes para a administração pública e que, indiretamente, afeta a população. O CTM temporal visa representar as mudanças e seus efeitos ao longo do tempo.

O método para a aquisição e organização dos dados temporais, baseado no processo de BI, utilizando Data Warehousing, se mostrou eficiente para a elaboração de relatórios, mapas e disponibilização de recursos que permitem ao usuário elaborar relatórios ad hoc, com dados em tabelas e gráficos. Dentre suas características, destaca-se a possibilidade de comparação entre dados atuais e dados históricos, demonstrando a evolução dos objetos ou, em alguns casos, ser fonte de controle de qualidade dos dados coletados em campo.

O STDW representa um novo campo de investigação, necessitando-se do desenvolvimento de uma abordagem metodológica consistente para aplicações em Cadastro e Gestão Territorial, portanto, sabe-se que a inclusão de dados espaciais aumenta o poder do processo decisório do gestor, por expandir o escopo da análise e por fornecer um realce, facilitando a visualização. Semelhantemente, o suporte temporal possibilita que os elementos de um modelo multidimensional sejam variáveis no tempo.

As análises, exemplificadas com o caso das cardiopatias e realizadas com os dados do STDW, demonstraram que modelos espaço-temporais permitem um entendimento das ocorrências registradas através do acompanhamento da evolução dos dados. Aos gestores são garantidas mais informações para apoiar suas decisões administrativas, por meio de relatórios, por meio gráfico e por meio dos mapas, sendo possível acompanhar e entender onde e quando os fenômenos acontecem.

\section{Agradecimentos}

À Universidade Estadual de Mato Grosso do Sul - UEMS pelo apoio, por meio do afastamento integral para estudo da doutoranda. À Fundação de Apoio ao Desenvolvimento do Ensino, Ciência e Tecnologia do Estado de Mato Grosso do Sul - FUNDECT pelo apoio, por meio da concessão da bolsa de estudo da doutoranda (DIÁRIO OFICIAL n. 7.719 PÁGINA 28, 02 DE JUNHO DE 2010. EDITAL DE HOMOLOGAÇÃO Chamada FUNDECT N ${ }^{\circ}$ 17/2009 - POSGRAD - Doutorado no País.). Ao Programa de Pós-Graduação em Ciências Cartográficas da FCT/UNESP de Presidente Prudente -SP, pelos laboratórios e apoio para o desenvolvimento da pesquisa. Ao Grupo de Pesquisa em Análise e Representação de Dados Espaciais - GARDE.

\section{Contribuição dos Autores}

Os autores Glaucia Gabriel Sass e Amilton Amorim contribuíram em todos os itens, desde a Conceitualização até a Redação - redação e revisão. Rui Pedro Julião contribuiu na Visualização, Redação minuta inicial e na Redação - revisão e edição.

\section{Conflitos de Interesse}

Os autores declaram que não há conflitos de interesse.

\section{Referências}

AILAMAKI, A.; et al. PostgreSQL. In.: SILBERSCHATZ, A.; KORTH, H. F.; SUDARSHAN, S. (Ed.). Sistemas de Banco de Dados. $5^{\text {a }}$ ed. São Paulo: Makron Books. 2006. pp. 653-672.

AMORIM. A.; PELEGRINA, M. A.; JULIÃO, R. P. S. P. M. Cadastro e gestão territorial: uma visão lusobrasileira para a implementação de sistemas de informação cadastral nos municípios. São Paulo: UNESP. 2018. 131p. 
ANDRIENKO, G. et al. Space, time and visual analytics. International Journal Geographical Information Science, v. 24, n. 10., p. 1577-1600, 2010.

BENNETT, R. et al. Cadastral Futures: Building a New Vision for the Nature and Role of Cadastres. International Federation of Surveyors, Article of the Month (June) 2011. Disponível em: <https://fp-hid1076751.testsider.dk/resources/monthly_articles/2011/june_2011/june_2011_bennett_rajabifard_et_al.pd f>. Acesso em: nov. 2011.

DALE, P. F.; MCLAUGHLIN. J. D. Land information management: an introduction with special reference to cadastral problems in third world countries. Reprinted (with correction). Oxford. Oxford University Press. 1990. 300p.

DATE, C. J. Introdução a Sistemas de Banco de Dados. Tradução: Vandenberg Dantas de Souza. $7^{a}$ ed. Americana. Rio de Janeiro: Campus, 2000. 803p.

DAVE, C. Historical records with PostgreSQL, temporal tables and SQL. 2015. Disponível em: <http://clarkdave.net/2015/02/historical-records-with-postgresql-and-temporal-tables-and-sql-2011/>. Acesso em: abr. 2016.

DEBELJAČKI, R.; BOŠNJAK, S.; ŠEREŠ, L. The management of temporal data in relational databases. In: INTERNATIONAL CONFERENCE ON APPLIED INTERNET AND INFORMATION TECHNOLOGIES, 2015, Serbia, Zrenjanin. Proceedings... Zrenjanin: University of Novi Sad, Technical Faculty "Mihajlo Pupin", 2015. p. 45-49.

EDELWEISS, N. Banco de Dados Temporais: Teoria e Prática. Jornada de Atualização em Informática. In: CONGRESSO NACIONAL DA SOCIEDADE BRASILEIRA DE COMPUTAÇÃO, XVII, Belo Horizonte. Anais... SBC. Belo Horizonte, 1998. pp. 225-282.

ELMASRI, R.; NAVATHE, S. B. Sistemas de banco de dados. Revisor: Luis Ricardo de Figueiredo. $4^{\mathrm{a}}$ ed. São Paulo: Pearson Addison Wesley. 2005. 728p.

FONSECA, R. L. et al. Um metamodelo para especificação de data warehouse geográfico. In: SIMPÓSIO BRASILEIRO DE BANCO DE DADOS, XXII, 2007, João Pessoa, Paraíba. Anais... Disponível em: <http://www.lbd.dcc.ufmg.br/bdbcomp/servlet/Trabalho?id=6949> Acesso em: ago. 2011.

INMON, W. H. Building the Data Warehouse. $4^{\mathrm{a}}$ ed. Indianapolis: Wiley Publishing. 2005. 428p.

JAKOBSSON, H.; ORACLE CORPORATION. Oracle. In: SILBERSCHATZ, A.; KORTH, H. F.; SUDARSHAN, S. (ed). Sistemas de Banco de Dados. $5^{\text {a }}$ ed. São Paulo: Makron Books, 2006. pp. 674691.

JENSEN, C. et al. The consensus glossary of temporal database concepts - February, 1998 version. Site <http://www.cs.aau.dk/ csj/Glossary/> Acesso em: jan. 2011.

JENSEN, C. S.; SNODGRASS, R. T. Temporal data management. IEEE Transactions on Knowledge and Data Engineering. v. 11, n. 1, p. 36-44, 1999.

KEIM, D. et al. Visual analytics: definition, process, and challenges. Information visualization - humancentered issues and perspectives. A. Kerren (ed); J.T. Stasko (ed); J. D. Fekete (ed); C. North(ed). Volume 4950 of LNCS state-of the-art survey. Berlin: Springer, 2008. pp. 154-175.

LIU, Q., et al. Permutation-test-based clustering method for detection of dynamic patterns in Spatiotemporal datasets. Computers, Environment and Urban Systems, v. 75, 2019, p. 204-216, ISSN 01989715. Disponível em: <https://doi.org/10.1016/j.compenvurbsys.2019.02.007> Acesso em: jun. 2019.

LONGLEY, P. A. et al. Sistemas e Ciência da Informação Geográfica. $3^{\circ}$ ed. Porto Alegre: Bookman, 2013. 3-35p.

MALINOWSKI, E.; ZIMÁNYI, E. Advanced data warehouse design: from conventional to spatial and temporal applications. 2nd corrected printing. Springer-Verlag Berlin Heidelberg, 2008. 362p.

MEDEIROS, C. B.; JOMIER, G. Managing alternatives and data evolution in GIS. In: WORKSHOP ON ADVANCES IN GEOGRAPHIC INFORMATION SYSTEMS, ACM/ISCA Baltimore, USA, November, 
1993. Proceedings... $\quad$ Baltimore, USA, 1993. p. $\quad 36-39 . \quad$ Site <http://citeseerx.ist.psu.edu/viewdoc/summary?doi=10.1.1.54.6261> Acesso em: maio 2011.

ORACLE CORP. Workspace Manager Developer's Guide 11g Release 2 (11.2). 2013. Disponível em: <https://docs.oracle.com/cd/E11882_01/appdev.112/e11826/title.htm> Acesso em: mar. 2016.

PGDG (PostgreSQL Global Development Group). Chapter 8: Data Types. PostgreSQL 9.5.2 Documentation. 2016. Disponível em: <http://www.postgresql.org/docs/9.5/static/datatype-datetime.html> Acesso em: abr. 2016.

PIUMETTO, M.; ERBA, D. A. Sistema de información geográfica aplicadas al catastro urbano. In: ERBA, D. A. (Ed). Cadastro Multifinalitario aplicado a la definición de políticas de suelo urbano. Cambridge, MA: Lincoln Institute of Land Policy, 2007. Cap 9. p. 242 - 266.

POLAT, Z. A., et al. A LADM-based temporal cadastral information system for modelling of easement rights - A case study of Turkey. Survey Review, 52:370, 2018. pp.1-12. DOI. 10.1080/00396265.2018.1503481

ROB, P.; CORONEL, C. Sistemas de banco de dados: projeto, implementação e gerenciamento. Tradução: All Tasks. Revisão Técnica: Ana Paula Appel. $8^{a}$ ed. norte-americana. São Paulo: Cengage Learning. 2011. $744 \mathrm{p}$.

SASS, G. G. Um Método de Análise de Dados Temporais para o Cadastro Territorial Multifinalitário Urbano. Tese de doutoramento. Faculdade de Ciência e Tecnologia da Universidade Estadual Paulista UNESP, Programa de Pós-Graduação em Ciências Cartográficas, Presidente Prudente - SP, 2013. 151p.

SASS, G. G.; AMORIM. A. Análise Temporal a Partir do Cadastro Territorial Multifinalitário. Revista Brasileira de Cartografia, v. 65, p. 283-291, 2013.

SHETH, A. Changing focus on interoperability in information systems: from system, syntax, structure to semantics. In: GOODCHILD, M.; EGENHOFER, M.; FEGEAS, R.; KOTTMAN, C. (Ed.) Interoperating geographic information systems. Kluwer Academic Publishers, Norwell, Ma. 1999. pp. 5-29.

SILBERSCHATZ, A.; KORTH, H. F.; SUDARSHAN, S. Sistema de Banco de Dados. $5^{\text {a }}$ ed. São Paulo: Makron Books. 2006.

SNODGRASS, R. T. (Ed.) The TSQL2 Temporal Query Language.Kluwer Academic. Publishers Northwell, MA. 1995. Disponível em: <https://www2.cs.arizona.edu/ rts/pubs/PODS84.pdf〉, Acesso em: maio 2012.

SNODGRASS, R. T.; GAO, D.; ZHANG, R.; THOMAS, S. W. Temporal Support for Persistent Stored Modules. In: IEEE INTERNATIONAL CONFERENCE ON DATA ENGINEERING, $28^{\text {th }}$, Washington, DC, 2012. Proceedings... pp. 114-125.

STEUDLER, DANIEL (ed). FIG Report: CADASTRE 2014 and Beyond. N. 61. Copenhagen, Denmark. May 2014. 84p. Disponível em: 〈https://fig.net/resources/publications/figpub/pub61/Figpub61.pdf> Acesso em: jul. 2016.

TRAJCEVSKI, G. et al. Semantics-Aware Warehousing of Symbolic Trajectories. In: ACM SIGSPATIAL INTERNATIONAL WORKSHOP ON GEOSTREAMING, 6th, 2015, New York. Proceedings... ACM, New York, USA, 2015. p. 1-8.

VAISMAN A.; ZIMÁNYI, E. Mobility Data Warehouses. ISPRS International Journal of GeoInformation. 8(4):170, 2019. DOI. 10.3390/ijgi8040170

VAISMAN, A.; ZIMÁNYI, E. What Is Spatio-Temporal Data Warehousing? In: INTERNATIONAL CONFERENCE ON DATA WAREHOUSING AND KNOWLEDGE DISCOVERY (DAWAK '09), $11^{\text {th }}$, 2009, Berlin, Heidelberg. Proceedings... Torben Bach Pedersen, Mukesh K. Mohania, and A Min Tjoa (Eds.). Springer-Verlag, Berlin, Heidelberg. 2009. p. 9-23.

YUAN, C., et al. Analysis of Surface Subsidence Caused by Excavation of Urban Tunnel Based on GIS. In: INTERNATIONAL CONFERENCE ON TRANSPORTATION INFRASTRUCTURE AND 
MATERIALS (ICTIM 2017), 2017, Qingdao, China. Proceedings... DOI. 10.12783/dtmse/ictim2017/9954

WILLIAMSON, I. et al. Land administration for sustainable development. Redlands - California, Esri Press. 2010. 512p.

\section{Biografia do autor principal}

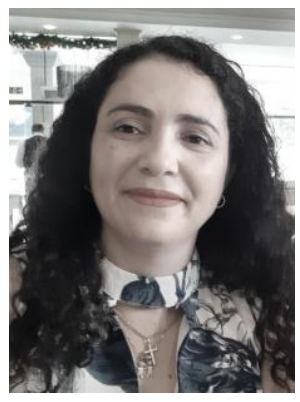

Glaucia Gabriel Sass nasceu em 1976 no município de Maringá, Pr. Possui graduação em Tecnologia em Processamento de Dados pela Faculdade de Administração e Informática de Maringá, mestrado em Informática pela Universidade Federal do Paraná e doutorado em Ciências Cartográfica pela UNESP - Presidente Prudente. Atualmente é professora titular da Universidade Estadual de Mato Grosso do Sul, atuando no ensino de graduação e pós-graduação. Está, desde marco/2020, como diretora da Diretoria de Informática da UEMS. Os interesses atuais de pesquisa envolvem Sistemas de Informação, Análises de Dados Espaciais e Temporais, Educação a Distância e Informática em Saúde.

Esta obra está licenciada com uma Licença Creative Commons Atribuição 4.0 Internacional - CC BY. Esta licença permite que outros distribuam, remixem, adaptem e criem a partir do seu trabalho, mesmo para fins comerciais, desde que lhe atribuam o devido crédito pela criação original. 